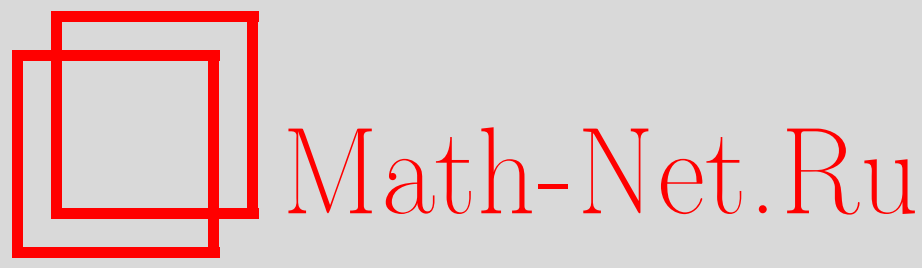

М. Б. Эпендиев, О 4-импульсе поля точечного заряда в нелинейной электродинамике, ТМФ, 2017, том 191, номер 3, 417-423

DOI: https://doi.org/10.4213/tmf9249

Использование Общероссийского математического портала Math-Net.Ru подразумевает, что вы прочитали и согласны с пользовательским соглашением http://www . mathnet.ru/rus/agreement

Параметры загрузки:

IP : 52.6 .47 .48

26 апреля 2023 г., $15: 27: 12$

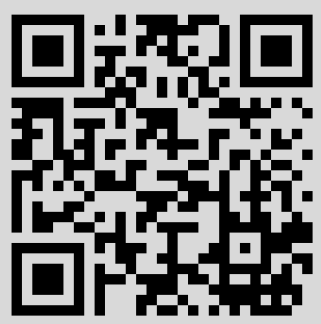




\title{
ФИЗИКА
}

Том 191, № 3

июнь, 2017

(C) 2017 г.

М. Б. Эпендиев*

\section{О 4-ИМПУЛЬСЕ ПОЛЯ ТОЧЕЧНОГО ЗАРЯДА В НЕЛИНЕЙНОЙ ЭЛЕКТРОДИНАМИКЕ}

\begin{abstract}
Доказано, что 4-импульс электромагнитного поля точечного заряда является 4-вектором, если лагранжиан поля нелинеен (относительно инвариантов поля), а масса поля конечна. Определен класс лагранжианов, приводящих к ограниченности массы поля.
\end{abstract}

Ключевые слова: нелинейная электродинамика, точечный заряд, масса поля, 4-импульс поля.

DOI: https://doi.org/10.4213/tmf9249

\section{1. ВВЕДЕНИЕ}

В данной работе принята система единиц, в которой скорость света $c=1$. Латинские индексы пробегают значения $0,1,2,3$; греческие - $1,2,3$. По повторяющимся (сверху и снизу) индексам проводится суммирование. Если в интегралах не указаны пределы интегрирования, то они бесконечны. Компонентами 4-вектора $w^{k}$ является совокупность $\left(w^{0}, \vec{w}\right)$; в частности, координатный вектор $x^{k}=(t, \vec{r})$, вектор 4-скорости $u^{k}=\left(u^{0}, \vec{u}\right)$. Иногда вектор записывается без указания индекса, причем $(x u)=x^{n} u_{n}, x^{2}=x^{n} x_{n}$, и т. д.

В работе рассматривается вопрос о том, в каких случаях 4-импульс поля можно считать 4-вектором. Чтобы избежать разночтений, вкратце напомним, что такое 4-вектор (и тензор вообще) в псевдоевклидовом пространстве.

В основе определения тензора лежит принцип релятивистской инвариантности: соотношения между тензорными выражениями не меняются при переходе из одной инерциальной системы отсчета в другую. При указанном переходе новые (штрихованные) и старые координаты связаны ортогональным преобразованием

$$
x^{\prime k}=Q_{i}^{k} x^{i}, \quad x^{k}=x^{i} Q_{i}{ }^{k}, \quad Q_{i}{ }^{k} Q_{n}^{i}=Q_{i}^{k} Q_{n}{ }^{i}=\delta_{n}^{k} .
$$

Совокупность величин $A_{k_{1} \ldots k_{s}}^{i_{1} \ldots i_{n}}(x)$ можно назвать тензором, если

$$
A_{k_{1} \ldots k_{s}}^{i_{1} \ldots i_{n}}\left(x^{\prime}\right)=Q_{l_{1}}^{i_{1}} \ldots Q_{l_{n}}^{i_{n}} Q_{k_{1}}^{j_{1}} \ldots Q_{k_{s}}^{j_{s}} A_{j_{1} \ldots j_{s}}^{l_{1} \ldots l_{n}}(x)
$$

(в правой части $(2) x=x\left(x^{\prime}\right)$ в соответствии с $\left.(1)\right)$.

* Институт машиноведения им. А. А. Благонравова РАН, Москва, Россия. E-mail: m010148@mail.ru 
Обычно под преобразованием (1) подразумевают или преобразование Лоренца (переход в движущуюся систему отсчета), или поворот пространственных осей. В общем случае выражение (1) описывает любую последовательность этих действий. В книге [1] дано представление матрицы $Q$ как экспоненты антисимметричной матрицы:

$$
\begin{aligned}
Q_{k}^{i}=\left(e^{W}\right)_{k}^{i}=\frac{1}{\theta}\left\{\left(\varphi^{2} \operatorname{ch} \gamma\right.\right. & \left.+\gamma^{2} \cos \varphi\right) \delta_{k}^{i}+(\gamma \operatorname{sh} \gamma+\varphi \sin \varphi) W_{k}^{i}+ \\
& \left.+(\operatorname{ch} \gamma-\cos \varphi) W_{n}^{i} W_{k}^{n}+(\varphi \operatorname{sh} \gamma-\gamma \sin \varphi) \widetilde{W}_{k}^{i}\right\},
\end{aligned}
$$

где

$$
\begin{array}{lll}
W_{k}^{i}=-W_{k}{ }^{i}, & \widetilde{W}^{i k}=\frac{1}{2} e^{i k l n} W_{l n} \quad\left(e^{0123}=1\right), & \theta=\sqrt{a_{0}^{2}+b_{0}^{2}}, \\
\gamma=\sqrt{\frac{\theta+a_{0}}{2}}, & \varphi=\frac{b_{0}}{2 \gamma}, \quad a_{0}=\frac{1}{2} W^{l n} W_{n l}, & b_{0}=\frac{1}{2} W^{l n} \widetilde{W}_{n l} .
\end{array}
$$

При $W_{\beta}^{\alpha}=0$ матрица (3) является оператором преобразования Лоренца, при $W_{0}^{\alpha}=0$ оператором поворота пространственных осей.

Если $T^{k n}(x)$ есть тензор энергии-импульса (ТЭИ) некоторой системы, то 4-импульс системы определяется интегралом

$$
P^{k}=\int T^{k 0} d^{3} \vec{r}
$$

Очевидно, что выражение (4) в общем случае не является 4-вектором. С другой стороны, если система изолирована, то (4) должно быть 4-вектором (иначе или данную систему нельзя изолировать, или 4-импульс не является ее динамической переменной). Мы собираемся обсуждать 4-импульс электромагнитного поля некоторого заряда. Ясно, что между зарядом и его полем существует некая связь, так как отделить (изолировать) поле от заряда невозможно. Одна из сторон этой связи выявлена в статье [2], где рассматривается взаимодействие ускоренно движущегося протяженного заряда со своим полем. Показано, что в первом приближении (но только в первом) при действии на заряд внешней силы инерционным параметром является сумма массы заряда и массы поля. Кроме того, протяженность заряда позволяет говорить о наличии неких внутренних процессов в нем и связать эти процессы со свойствами поля так, чтобы 4-импульс системы "заряд+поле" являлся 4-вектором. Для точечного же заряда 4-импульс по определению является 4-вектором. Однако при линейном (относительно инвариантов поля) лагранжиане энергия его поля расходится. Поэтому в данном случае, чтобы понятие 4-импульса имело физический смысл, нужно исходить из нелинейных лагранжианов.

Нелинейные поправки обычно рассчитываются в квантовой теории при рассмотрении радиационных процессов (см. книгу [3]). Мы ограничиваемся в настоящей статье зависимостью лагранжиана от одного инварианта поля, но зато исследуем эту зависимость в общем виде. 


\section{2. ЭНЕРГИЯ И ИМПУЛЬС ПОЛЯ}

Лагранжиан, уравнение и ТЭИ поля имеют вид $\left(J^{k}-\right.$ вектор тока)

$$
\begin{gathered}
L=L(a), \quad a=\frac{1}{4} F^{k n} F_{n k}, \quad F_{k n}=A_{n, k}-A_{k, n}, \\
\left(L^{\prime} F^{k n}\right)_{, n}=-J^{k}, \quad(\cdot)_{, n}=\frac{\partial}{\partial x^{n}}(\cdot), \\
T^{k n}=L^{\prime} F^{k m} F_{m}^{. n}-g^{k n} L, \quad L^{\prime}=\frac{d L}{d a} .
\end{gathered}
$$

Для покоящегося заряда и сферической симметрии

$$
\begin{gathered}
J^{k}=(\mu(r), 0,0,0), \quad A^{k}=(\Phi(r), 0,0,0), \quad a=\frac{1}{2} f^{2}, \quad f=\frac{d \Phi}{d r}, \\
F_{(0)}^{0 \alpha}=\frac{r^{\alpha} f}{r}, \quad F_{(0)}^{\alpha \beta}=0, \quad T_{(0)}^{00}=L^{\prime} f^{2}-L, \quad T_{(0)}^{0 \alpha}=0 .
\end{gathered}
$$

Из формул (5), (6), учитывая, что $\vec{\nabla}\left(\vec{r} / r^{3}\right)=4 \pi \delta(\vec{r})$, получаем уравнение

$$
4 \pi Q \delta(\vec{r})+\frac{1}{r^{2}} \frac{d Q}{d r}=-\mu, \quad Q=Q(r)=L^{\prime} r^{2} f .
$$

Отсюда следует, что

- если $\mu=e \delta(\vec{r})$, то

$$
Q=\text { const }=-\frac{e}{4 \pi}
$$

- если заряд "размазан", то $Q(0)=0$ и

$$
Q=-\int_{0}^{r} \tau^{2} \mu(\tau) d \tau
$$

Если заряд движется со скоростью $\vec{v}$, то равенства (5) переходят в равенства

$$
\begin{gathered}
F^{k n}=\frac{\left(q^{k} u^{n}-q^{n} u^{k}\right) f(\sigma)}{\sigma}, \\
q^{k}=(x u) u^{k}-x^{k}, \quad \sigma=\sqrt{(x u)^{2}-x^{2}}=|\vec{\sigma}|, \\
\vec{\sigma}=\vec{r}-\left(t-\frac{\vec{u} \vec{r}}{u_{0}+1}\right) \vec{u}, \quad d^{3} \vec{r}=\frac{d^{3} \vec{\sigma}}{u_{0}}, \quad \vec{u}=u_{0} \vec{v}, \quad u_{0}=\sqrt{1+\vec{u}^{2}}, \\
T^{k n}=\left(u^{k} u^{n}-\frac{q^{k} q^{n}}{\sigma^{2}}\right) L^{\prime} f^{2}-g^{k n} L, \quad f=f(\sigma), \quad a=\frac{f^{2}}{2} .
\end{gathered}
$$


Обозначим

$$
\langle Q(\vec{r})\rangle=\int Q(\vec{r}) d^{3} \vec{r},
$$

причем для любой интегрируемой функции $B(\vec{r})$

$$
\int B(\vec{\sigma}(\vec{r})) d^{3} \vec{r}=\langle B(\vec{\sigma}(\vec{r}))\rangle=\frac{1}{u_{0}}\langle B(\vec{r})\rangle .
$$

Учитывая, что

$$
q^{0}=-(\vec{u} \vec{\sigma}), \quad \vec{q}=-\vec{\sigma}-\frac{(\vec{u} \vec{\sigma}) \vec{u}}{u_{0}+1}, \quad \vec{r}=\vec{\sigma}+\frac{\vec{u}}{u_{0}}\left(t-\frac{(\vec{u} \vec{\sigma})}{u_{0}+1}\right),
$$

а также то, что для всякой интегрируемой функции $Q(\sigma)$ имеет место соотношение

$$
\left\langle\sigma_{\alpha} \sigma_{\beta} Q(\sigma)\right\rangle=\frac{\delta_{\alpha \beta}}{3}\left\langle\sigma^{2} Q(\sigma)\right\rangle, \quad \delta_{\alpha \alpha}=1, \quad \alpha \neq \beta \rightarrow \delta_{\alpha \beta}=0,
$$

для компонент 4-импульса поля из формулы (4) получаем выражения

$$
\begin{aligned}
& P_{f}^{0}=\left\langle T^{00}\right\rangle=\left\langle\left(u_{0}-\frac{\vec{u}^{2}}{3 u_{0}}\right) L^{\prime} f^{2}-\frac{L}{u_{0}}\right\rangle, \\
& P_{f}^{\alpha}=\left\langle T^{0 \alpha}\right\rangle=\left\langle L^{\prime} f^{2}\right\rangle \frac{2 u^{\alpha}}{3}, \quad f=f(r) .
\end{aligned}
$$

Вводя массу поля

$$
M_{f}=\left\langle T_{(0)}^{00}\right\rangle=\left\langle L^{\prime} f^{2}-L\right\rangle, \quad f=f(r),
$$

перепишем (11) в виде

$$
P_{f}^{k}=M_{f} u^{k}+C b^{k}
$$

где

$$
b^{0}=\frac{\vec{u}^{2}}{3 u_{0}}, \quad b^{\alpha}=\frac{u^{\alpha}}{3}, \quad b^{n} u_{n}=0, \quad C=\left\langle 3 L-L^{\prime} f^{2}\right\rangle, \quad f=f(r) .
$$

Так как $b^{k}$ не является 4-вектором, необходимым и достаточным условием того, что (13) представляет собой 4-вектор, является равенство $C=0$ при ограниченной мacce $M_{f}$.

При линейном лагранжиане $(L=a / 4 \pi) C=\left\langle f^{2}\right\rangle / 8 \pi \neq 0$. В то же время для ограниченности массы (а также и $C$ ) нужно, чтобы заряд был протяженным.

Далее мы рассматриваем поле точечного заряда, считая, что лагранжиан нелинейный и такой, что интеграл (12) не расходится. Тогда из (9) имеем равенства

$$
L^{\prime} f=-\frac{e}{4 \pi r^{2}}, \quad f=-\frac{e \sqrt{2 a}}{|e|} .
$$

Отсюда находим $a=a(r)$ или $r=r(a)$ :

$$
r=r(a)=\frac{\beta_{0}}{\sqrt{z}}, \quad \beta_{0}=\sqrt{\frac{|e|}{\sqrt{2} 4 \pi}}, \quad z=\sqrt{a} L^{\prime}
$$


Тогда для функций вида $B(a, r)$ записываем формулу

$$
\langle B\rangle=2 \pi \beta_{0}^{3} \int_{0}^{a_{(0)}} B(a, r(a)) \frac{z^{\prime}}{z^{5 / 2}} d a, \quad z^{\prime}=\frac{d z}{d a}, \quad 2 a_{(0)}=(f(0))^{2}
$$

$\left(a_{(0)}\right.$ есть значение $a$, при котором $\left.L^{\prime}=\infty\right)$.

В ряде случаев преобразование (16) может приводить к более простым интегралам. Так, интегралы (43), (44), вычисленные в работах [4], [5], с помощью этого преобразования можно выразить через гамма-функцию:

$$
I_{1}=\frac{2(\Gamma(1 / 4))^{2}}{3^{9 / 4} \sqrt{2 \pi}} \cong 0.8855, \quad I_{2}=\frac{9 I_{1}}{2} \cong 3.9847 .
$$

При этом в выражении для массы (находящемся сразу после (44)) коэффициент 2.65 можно заменить на $3 I_{1} \cong 2.6565$.

Вместо (14) для случая (15) с учетом (16) и (12) получаем, что

$$
\begin{aligned}
C & =2 \pi \beta_{0}^{3} \int_{0}^{a_{0}}\left(3 L-L^{\prime} f^{2}\right) \frac{z^{\prime}}{z^{5 / 2}} d a= \\
& =4 \pi \beta_{0}^{3} \int_{0}^{a_{0}} \frac{d}{d a}\left(\frac{2 L^{\prime} a-L}{z^{3 / 2}}\right) d a=4 \pi \int_{0}^{\infty} \frac{d}{d r}\left(r^{3} T_{(0)}^{00}\right) d r .
\end{aligned}
$$

Если плотность энергии удовлетворяет условиям

$$
\lim _{r \rightarrow 0} r^{3} T_{(0)}^{00}=0, \quad \lim _{r \rightarrow \infty} r^{3} T_{(0)}^{00}=0,
$$

то приходим к тождеству

$$
C=\left\langle 3 L-2 L^{\prime} a\right\rangle \equiv 0 .
$$

Из ограниченности выражения (12) следуют условия (18) (обратное, вообще говоря, неверно). Отметим, что согласно (19) 4-импульс (13) переходит для случая точечного заряда в 4-вектор, введенный в работе [4] в качестве вектора энергии-импульса.

Таким образом, 4-импульс (13) есть 4-вектор, если заряд точечный, а нелинейность лагранжиана такова, что масса поля конечна. В других случаях (размазанность заряда, нарушение сферической симметрии и пр.) мы, вообще говоря, не вправе делать такое утверждение. А реальность и представляет собой такие "другие случаи". Поэтому подобно вырождению спектра в квантовой механике тождество (19) можно назвать случайным порождением точечной идеализации заряда.

Отметим, что в случае (19)

$$
M_{f}=2\langle L\rangle=\frac{4}{3}\left\langle L^{\prime} a\right\rangle=\frac{8 \pi \beta_{0}^{3}}{3} \int_{0}^{a_{(0)}} \frac{z^{\prime} \sqrt{a}}{z^{3 / 2}} d a .
$$

\section{3. УСЛОВИЯ ОГРАНИЧЕННОСТИ МАССЫ ПОЛЯ}

Рассмотрим, какие условия нужно наложить на функцию $L(a)$, чтобы интеграл в (20) сходился. Запишем этот интеграл в виде

$$
\int_{0}^{a_{(0)}} z^{\prime} \sqrt{a} \frac{d a}{z^{3 / 2}}=-\left.2 \sqrt{\frac{\sqrt{a}}{L^{\prime}}}\right|_{0} ^{a_{(0)}}+\int_{0}^{a_{(0)}} \frac{d a}{\sqrt{L^{\prime} a^{3 / 2}}} .
$$


Из (15) следует, что

$$
L^{\prime}=\frac{d L}{d a} \geqslant 0 \quad \text { при всех } \quad 0 \leqslant a \leqslant a_{(0)}
$$

Для ограниченности правой части (21), прежде всего, необходимо, чтобы

$$
\left.L^{\prime}\right|_{a \rightarrow 0} \sim a^{1 / 2-\delta_{1}} .
$$

Третье условие зависит от того, расходится поле в нуле $\left(a_{(0)}=\infty\right)$ или нет. При $a_{(0)}=\infty$ имеем

$$
\left.L^{\prime}\right|_{a \rightarrow \infty} \sim a^{1 / 2+\delta_{2}}
$$

(в конце заключения работы [4] упомянуто это условие). А при $a_{(0)}<\infty$

$$
\left.L^{\prime}\right|_{a \rightarrow a_{(0)}} \sim\left(C_{1}+C_{2}\left(a_{(0)}-a\right)^{\delta_{3}}\right)^{-2}, \quad C_{1}=\text { const } \geqslant 0, \quad C_{2}=\text { const } .
$$

В $(19)-(21) \delta_{1}, \delta_{2}, \delta_{3}-$ некоторые положительные константы, знак $\sim$ означает, что в (23) левая часть убывает медленнее правой, а в (24), (25) - левая часть растет быстрее правой.

$\mathrm{У}$ нас нет гарантий того, что тот или иной используемый лагранжиан является точным. Соответственно и расчет массы нельзя считать точным. В этом плане выделяются лагранжианы, при которых поле в нуле остается бесконечным. Это означает, что поправки к ним могут приводить к существенно другой массе. Продемонстрируем этот факт на простом примере.

Используемый в статьях [4], [5] лагранжиан

$$
L_{1}=\frac{a+\sigma_{0} a^{2} / 2}{4 \pi}, \quad a_{(0)}=\infty,
$$

можно считать суммой двух первых членов разложения лагранжиана

$$
L=-\frac{1}{4 \pi \sigma_{0}} \ln \left(1-\sigma_{0} a\right), \quad a_{(0)}=\frac{1}{\sigma_{0}},
$$

по относительно малому параметру $\sigma_{0}$. При этом отношение масс, рассчитанных для первого и второго вариантов, равно

$$
\frac{M_{f}\left(L_{1}\right)}{M_{f}(L)}=\frac{3 \Gamma(1 / 4) \Gamma(3 / 4)}{2 \pi} \cong 2.1213 .
$$

Однако, по мнению автора, такой возможный разброс массы поля в данной модели не принципиален, так как нам все равно не известно достаточно точное значение массы "голого" заряда. Инерционным параметром системы “заряд+поле" является суммарная масса, определенная для стабильных заряженных частиц с большой точностью. Поэтому для придания модели физического смысла достаточно убедиться, что в этой модели масса поля конечна и не превышает суммарную массу.

Благодарности. В заключение автор выражает искреннюю благодарность профессору МГУ им. М. В. Ломоносова А. В. Борисову за помощь и консультации в работе. 


\section{Список литературы}

[1] М. Б. Эпендиев, Теоретические основы физики, ИКИ, М.-Ижевск, 2013.

[2] М. Б. Эпендиев, "Классическая электродинамика ускоренно движущегося протяженного заряда", Вестн. Моск. ун-та. Сер. 3. Физ. Астрон., 6 (2014), 40-45; М. В. Ependiev, "On the foundations of the classical relativistic theory of the field of an accelerated extended charge", J. Modern Phys., 6 (2015), 601-609.

[3] В. Б. Берестецкий, Е. М. Лифшиц, А.П. Питаевский, Квантовая электродинамика, Наука, М., 1980.

[4] D. M. Gitman, A. E. Shabad, A. A. Shishmarev, Moving point charge as a soliton in nonlinear electrodynamics, arXiv: 1509.06401.

[5] C. V. Costa, D. M. Gitman, A. E. Shabad, "Finite field-energy of a point charge in QED", Phys. Scr., 90:7 (2015), 074012, 6 pp., arXiv: 1312.0447.

Поступила в редакцию 23.06.2016, после доработки 13.09.2016 